Taking the average over $t$ and once again utilizing (A2) we obtain

$$
\begin{aligned}
D^{\prime \prime} & =\int_{0}^{1} E \epsilon^{\prime \prime}(t)^{2} d t=\int \sum_{\nu}\left|G_{\nu}(\lambda)\right|^{2} \Phi_{W}(\lambda-\nu) d \lambda \\
& =\int G(\lambda) Q^{\prime \prime}(\lambda) G^{*}(\lambda) d \lambda
\end{aligned}
$$

where in the last step we have introduced the diagonal matrix

$$
\begin{aligned}
& Q^{\prime \prime}(\lambda) \triangleq\left\{Q_{i j}^{\prime \prime}(\lambda)\right\} \\
& Q_{i j}^{\prime \prime}(\lambda) \triangleq \delta_{i j} \Phi_{W}(\lambda-i) .
\end{aligned}
$$

If we finally define

$$
Q(\lambda) \triangleq Q^{\prime}(\lambda)+Q^{\prime \prime}(\lambda)
$$

and add the expressions (A3) and (A4), we obtain the desired result (3.7)

\section{REFERENCES}

[1] T. Berger and D. W. Tufts, "Optimum pulse amplitude modulation: part I," IEEE Trans. Inform. Theory, vol. IT-13, pp. 196-208, Apr. 1967.

[2] T. Berger, Rate Distortion Theory. Englewood Cliffs, NJ: Prentice Hall, 1971, pp. 151- 165 .

[3] H. L. van Trees, Detection, Estimation, and Modulation Theory: Part I. New York: Wiley, 1968, ch. 6.

[4] T. Ericson, "Optimum amplitude modulation," Univ. Linköping, Linköping, Sweden, Rep. LiTH-ISY-R-0040. 1974.

[5] E. Wong, Stochastic Processes in Information and Dynamical Systems. New York: McGraw-Hill, 1971.

[6] W. A. Gardner and L. E. Franks, "Characterization of cyclostationary random signal processes," IEEE Trans. Inform. Theory, vol IT-21, no. 1, pp. 4-14, Jan. 1975.

[7] W. R. Bennet, "Statistics of regenerative digital transmission," Bell Syst. Tech.J., vol. 37, pp. 1501-1542, Nov. 1958.

\title{
Tables of Sphere Packings and Spherical Codes
}

\author{
N. J. A. SLOANE, FELLOW, IEEE
}

\begin{abstract}
The theta function of a sphere packing gives the number of centers at each distance from the origin. The theta functions of a number of important packings ( $A_{n}, D_{n}, E_{n}$, the Leech lattice, and others) and tables of the first fifty or so of their coefficients are given in this paper.
\end{abstract}

\section{SUMmary}

$\mathrm{T}$ HE MAIN RESULTS in this paper are formulas for the theta functions of the sphere packings $A_{2}$, given in (30), $A_{n}$ (34), the face-centered cubic lattice $D_{3}(40)$, the body-centered cubic lattice $D_{3}^{\perp}(41)$, the hexagonal closepacking (42), $D_{n}(40), D_{n}^{\perp}(41), E_{6}(43), E_{7}(44), E_{8}(45)$, $K_{12}(49), \Lambda_{16}(51)$, and the Leech lattice $\Lambda_{24}(54)$. Tables I-X give the first fifty or so coefficients of these theta functions and are far more extensive than any tables hitherto published. (Some earlier tables were given in [4] and [44]; see also [40] and [41].)

Manuscript received March 26, 1980.

The author is with Bell Laboratories, Room 2C-363, Murray Hill, NJ 07974.
Spherical codes are defined in Section II, and one of the motivations for constructing these tables is that they supply excellent examples of spherical codes. The maximum inner product of any one of these codes is given by (12) and (13). Sections III-IX deal with the general properties of sphere packings and the associated spherical codes, while Section $\mathrm{X}$ gives formulas for the number of centers inside or on a large spherical shell. The connections with number theory are sketched in Section XI, and then Sections XII-XX give the most important packings in greater detail (including their generator matrices, densities and kissing numbers).

A sequel to this paper will discuss the encoding and decoding of these spherical codes and the nearest neighbor regions associated with the codewords.

\section{SPherical Codes}

Just as a binary error-correcting code [36] is a subset of the vertices of an $n$-dimensional cube, so a spherical code [16], [21] is a subset of the points of an $n$-dimensional sphere. More precisely, let $\Omega_{n}$ denote the unit sphere in $\boldsymbol{R}^{n}$, 
i.e. the points $\boldsymbol{x}=\left(x_{1}, \cdots, x_{n}\right) \in \boldsymbol{R}^{n}$ with $^{1}$

$$
\|\boldsymbol{x}\|:=x_{1}^{2}+\cdots+x_{n}^{2}=1 \text {. }
$$

A spherical code $C$ of dimension $n$, size $M$ and maximum dot product $s$ is a set of $M$ points of $\Omega_{n}$ with the property that

$$
x \cdot y \leqslant s \quad \text { for all } x, y \in C, x \neq y,
$$

where

$$
\boldsymbol{x} \cdot \boldsymbol{y}:=x_{1} y_{1}+\cdots+x_{n} y_{n} .
$$

The problem of finding the largest spherical code with a given dot product has a long history, especially in the case $n=3$ [13], [23]. Spherical codes have been extensively studied in connection with the design of signals for the Gaussian channel [3]-[6], [8], [31], [50], [55], [60], [63]. They also have applications to the design of quantizers and samplers [18], [19], [39], [58], in numerical quadrature [22], [56], tomography [49], and above all to diophantine equations (see Section XI). The theory of these codes borrows heavily from group representations, modular forms, and harmonic analysis [24], [27], [29], [35], [45], [46]. Spherical codes may be efficiently constructed from sphere packings, as we now demonstrate.

\section{Sphere Packings}

Stated informally, a sphere packing in $\boldsymbol{R}^{n}$ is an arrangement of infinitely many nonoverlapping spheres, all of the same size. More precisely, a sphere packing $\Lambda$ in $\boldsymbol{R}^{n}$ of radius $\rho$ consists of an infinite sequence of points $\boldsymbol{x}^{(1)}, \boldsymbol{x}^{(2)}, \cdots$ in $\dot{R}^{n}$ (the centers of the spheres) such that

$$
\operatorname{dist}\left(x^{(i)}, x^{(j)}\right)^{2}=\left\|x^{(i)}-x^{(j)}\right\| \geqslant 4 \rho^{2}
$$

for all $i \neq j$. Then if spheres of radius $\rho$ are drawn around the centers $x^{(1)}, x^{(2)}, \cdots$ the spheres will not overlap. $\Lambda$ is called a lattice packing if the centers $x^{(i)}$ form a group under componentwise addition. The literature on sphere packings is extensive, and the subject is intimately related to the theory of error-correcting codes. For more information the reader is referred to [1], [15], [34], [47], [48], [51]-[54].

\section{The Parameters of a Sphere Packing}

The dimension $d$ of a lattice packing $\Lambda$ is the maximum number of linearly independent centers in $\Lambda$. Since $\Lambda \subseteq \boldsymbol{R}^{n}$, $d \leqslant n$. Let $x^{(1)}=\left(x_{1}^{(1)}, \cdots, x_{n}^{(1)}\right), \cdots, x^{(d)}=\left(x_{1}^{(d)}, \cdots, x_{n}^{(d)}\right)$ $\in \Lambda$ be linearly independent centers which span the lattice. The $d \times n$ matrix

$$
M:=\left(x_{j}^{(i)}\right), \quad 1 \leqslant i \leqslant d, 1 \leqslant j \leqslant n,
$$

is a generator matrix for $\Lambda$, and $\Lambda$ consists of all integer combinations of the rows of $M$. The determinant of $\Lambda$ is

$$
\operatorname{det} \Lambda:=\left(\operatorname{det} M M^{T}\right)^{1 / 2} \text {. }
$$

When the lattice has the same dimension as the space in which it lies, i.e., when $d=n$, as is usually the case for the packings we shall consider, $M$ is a square matrix and

$$
\operatorname{det} \Lambda=|\operatorname{det} M| \text {. }
$$

The density $\Delta$ of any (lattice or nonlattice) sphere packing is, loosely speaking, the fraction of the space $\boldsymbol{R}^{n}$ that is covered by the spheres. For a lattice packing $\Lambda$ of radius $\rho$ and dimension $d=n$, the density is given by the formula

$$
\Delta=\frac{V_{n} \rho^{n}}{\operatorname{det} \Lambda}
$$

where

$$
V_{n}:=\frac{\pi^{n / 2}}{\Gamma((n / 2)+1)}
$$

is the volume of the unit sphere $\Omega_{n}$. The density of a nonlattice packing must be defined in a more complicated way-see [47]. The main sphere packing problem is to determine the sphere packings in $\boldsymbol{R}^{n}$ with the greatest density. References [34], [52], and [54] give the most recent results.

The kissing number $\tau(\boldsymbol{x})$ of the sphere centered at $\boldsymbol{x}$ is the number of neighboring spheres, i.e., the number of spheres which just kiss the sphere centered at $\boldsymbol{x}$ (using a term borrowed from billiards). The maximum value of $\tau(x)$ for $x \in \Lambda$ is denoted by $\tau_{\max }$. For a lattice packing $\tau(x)=$ $\tau_{\max }=\tau$, independently of the choice of $x$. A second important problem is to find the packings in $\boldsymbol{R}^{n}$ with the greatest value of $\tau_{\max }$ [2], [34], [43], [52].

\section{An Example: The Lattice $D_{4}$}

The lattice packing $D_{4}$ (see Section XVI) is a fourdimensional lattice in $R^{4}$ spanned by the vectors

$$
\begin{aligned}
& x^{(1)}=\frac{1}{\sqrt{2}}(2,0,0,0), \\
& x^{(2)}=\frac{1}{\sqrt{2}}(1,1,0,0), \\
& x^{(3)}=\frac{1}{\sqrt{2}}(1,0,1,0), \\
& x^{(4)}=\frac{1}{\sqrt{2}}(1,0,0,1) .
\end{aligned}
$$

Thus

$$
M=\frac{1}{\sqrt{2}}\left(\begin{array}{cccc}
2 & 0 & 0 & 0 \\
1 & 1 & 0 & 0 \\
1 & 0 & 1 & 0 \\
1 & 0 & 0 & 1
\end{array}\right)
$$

is a generator matrix, and $\operatorname{det} D_{4}=|\operatorname{det} M|=1 / 2$. The lattice points closest to the origin are the 24 points

$$
\begin{aligned}
& \frac{1}{\sqrt{2}}( \pm 1, \pm 1,0,0), \frac{1}{\sqrt{2}}( \pm 1,0, \pm 1,0), \frac{1}{\sqrt{2}}( \pm 1,0,0, \pm 1), \\
& \frac{1}{\sqrt{2}}(0, \pm 1, \pm 1,0), \frac{1}{\sqrt{2}}(0, \pm 1,0, \pm 1), \frac{1}{\sqrt{2}}(0,0, \pm 1, \pm 1),
\end{aligned}
$$

${ }^{1}$ Note that this definition of $\|x\|$ is the square of the usual one. 
so we may take the radius of the spheres to be $\rho=1 / 2$. or

The kissing number is $\tau=24$, and the density is

$$
\Delta=\frac{\pi^{2}}{16}=0.61686 \cdots
$$

\section{The Theta Function of a Lattice}

Many properties of a sphere packing can be obtained from its theta function, which is analogous to the weight enumerator of a code [36, ch. 5] in that it gives the number of centers at each distance from the origin. There is one important difference, however: a weight enumerator is a polynomial while a theta function is an infinite sum.

Definition: The theta function of a sphere packing $\Lambda$ is

$$
\Theta_{\Lambda}(z):=\sum_{x \in \Lambda} q^{\|x\|}
$$

where $q=e^{\pi i z}$. If $\Lambda$ is a lattice packing, as it usually is in this paper, the theta function is a holomorphic function of $z$ for $\operatorname{Im}(z)>0$ (see [24, p. 71]).

If $N_{m}$ denotes the number of centers $\boldsymbol{x} \in \Lambda$ with $\|\boldsymbol{x}\|=m$, i.e., at a squared distance of $m$ from the origin, then (7) can be rewritten as

$$
\Theta_{\Lambda}(z)=\sum_{m=0}^{\infty} N_{m} q^{m}
$$

where $m$ runs through all the values of $\|x\|$ for $x \in \Lambda$. The first two terms are $\Theta_{\Lambda}(z)=1+\tau q^{4 \rho^{2}}+\cdots$, where $\rho$ is the radius of $\Lambda$ and $\tau=\tau(0)$ is the kissing number of the sphere at the origin. For example the theta function of $D_{4}$ begins $\Theta_{D_{A}}(z)=1+24 q+24 q^{2}+96 q^{3}+\cdots$ (see Table V).

If $\Lambda$ is a lattice packing in $R^{n}$ of dimension $n$, the dual lattice $\Lambda^{\perp}$ is defined to be $\Lambda^{\perp}:=\left\{\boldsymbol{x} \in \boldsymbol{R}^{n} \mid \boldsymbol{x} \cdot \boldsymbol{y} \in \boldsymbol{Z}\right.$ for all $y \in \Lambda$. A generator matrix for $\Lambda^{\perp}$ is $\left(M^{-1}\right)^{\mathrm{tr}}$, and its determinant and theta function are

$$
\begin{aligned}
\operatorname{det} \Lambda^{\perp} & =(\operatorname{det} \Lambda)^{-1} \\
\Theta_{\Lambda^{\perp}}(z) & =(\operatorname{det} \Lambda)\left(\frac{i}{z}\right)^{n / 2} \Theta_{\Lambda}\left(-\frac{1}{z}\right) .
\end{aligned}
$$

\section{RESCALING}

It is frequently necessary to rescale a sphere packing, replacing $\Lambda$ by $\Lambda^{\prime}=c \Lambda=\{c x: x \in \Lambda\}$ for some appropriate constant $c \in \boldsymbol{R}$. The parameters of $\Lambda^{\prime}$ and $\Lambda$ are related as follows:

$$
\begin{aligned}
\rho^{\prime} & =c \rho, \\
\operatorname{dim} \Lambda^{\prime} & =\operatorname{dim} \Lambda, \\
M^{\prime} & =c M, \\
\operatorname{det} \Lambda^{\prime} & =c^{\operatorname{dim} \Lambda} \cdot \operatorname{det} \Lambda, \\
\left(\Lambda^{\prime}\right)^{\perp} & =\frac{1}{c} \Lambda^{\perp}, \\
\Delta^{\prime} & =\Delta, \\
\tau^{\prime}(c \boldsymbol{x}) & =\tau(\boldsymbol{x}), \quad \tau^{\prime}=\tau, \\
\Theta_{\Lambda^{\prime}}(z) & =\Theta_{\Lambda}\left(c^{2} z\right),
\end{aligned}
$$

$$
\Theta_{\Lambda^{\prime}}(z)=\left.\Theta_{\Lambda}(z)\right|_{\text {replace } q \text { by } q^{c^{2}}}
$$

\section{JACOBI Theta Functions}

The theta functions of many packings can be specified concisely in terms of the classical Jacobi theta functions $\theta_{2}$, $\theta_{3}$, and $\theta_{4}$, which are defined as follows: ${ }^{2}$

$$
\begin{aligned}
\theta_{2}(z): & =2 \sum_{m=0}^{\infty} q^{(m+(1 / 2))^{2}} \\
& =2 q^{1 / 4}+2 q^{9 / 4}+2 q^{25 / 4}+\cdots \\
& =2 q^{1 / 4}\left(1+q^{2}+q^{6}+q^{12}+q^{20}+\cdots\right), \\
\theta_{3}(z): & =1+2 \sum_{m=1}^{\infty} q^{m^{2}} \\
& =1+2 q+2 q^{4}+2 q^{9}+\cdots \\
& =1+2 q\left(1+q^{3}+q^{8}+q^{15}+\cdots\right), \\
\theta_{4}(z): & =1+2 \sum_{m=1}^{\infty}(-q)^{m^{2}} \\
& =1-2 q+2 q^{4}-2 q^{9}+\cdots \\
& =1-2 q\left(1-q^{3}+q^{8}-q^{15}+\cdots\right) .
\end{aligned}
$$

It is important to notice that $\theta_{3}$ is itself the theta function of the one-dimensional lattice of integer points, $Z$, in $\boldsymbol{R}^{1}$ :

$$
\theta_{3}(z)=\Theta_{Z}(z)
$$

and that

$$
\theta_{2}(z)=\Theta_{Z+(1 / 2)}(z)
$$

Furthermore,

$$
\theta_{4}(z)=\theta_{3}(z+1)
$$

We also point out that these theta functions can be written as infinite products:

$$
\begin{aligned}
& \theta_{2}(z)=2 q^{1 / 4} \prod_{m=1}^{\infty}\left(1-q^{2 m}\right)\left(1+q^{2 m}\right)^{2}, \\
& \theta_{3}(z)=\prod_{m=1}^{\infty}\left(1-q^{2 m}\right)\left(1+q^{2 m-1}\right)^{2}, \\
& \theta_{4}(z)=\prod_{m=1}^{\infty}\left(1-q^{2 m}\right)\left(1-q^{2 m-1}\right)^{2} .
\end{aligned}
$$

There are many other useful identities relating these functions-see [45], [46], [52, eqs. (14)-(23)], [53, eqs. (9)-(19)], [57], [62].

Example: The theta function of $D_{4}$ is (see Section XVI)

$$
\Theta_{D_{4}}(z)=\frac{1}{2}\left(\theta_{3}\left(\frac{z}{2}\right)^{4}+\theta_{4}\left(\frac{z}{2}\right)^{4}\right)
$$

\footnotetext{
${ }^{2}$ In the older literature these functions are denoted by $\theta_{2}(0 \mid z), \theta_{3}(0 \mid z)$, and $\theta_{4}(0 \mid z)$, but except in Section XV we simply omit the first argument (see also $[46$, sec. 7.5$],[62$, ch. XXI]).
} 


\section{CONSTRuction of Spherical CODES from SPHERE PACKINGS}

The construction is very simple. Suppose there are $N_{m}$ centers $x$ with $\|x\|=m$ in a sphere packing $\Lambda$. Then these points, rescaled by dividing them by $\sqrt{m}$, form a spherical code of dimension $n$ and size $N_{m}$. In other words we take a shell of points around the origin as the spherical code. The theta function of $\Lambda$ is thus a generating function for the sizes of these codes.

To find the maximum dot product $s$ of one of these codes we may either rescale the points and use (1), or work directly with the centers of $\Lambda$ and replace (1) by

$$
\begin{aligned}
s & =\max _{\boldsymbol{x} \neq \boldsymbol{y}} \cos \measuredangle(\boldsymbol{x} \mathbf{0} \boldsymbol{y}) \\
& =\max _{\boldsymbol{x} \neq \boldsymbol{y}} \frac{\boldsymbol{x} \cdot \boldsymbol{y}}{m}
\end{aligned}
$$

taken over distinct centers $x, y$, in the shell. It is easy to find $s$. Suppose $2 r$ is the smallest distance between any two points in the code. Then

$$
s=1-\frac{2 r^{2}}{m}
$$

- see Fig. 1. Certainly $r$ cannot be less than $\rho$, the radius of the spheres in $\Lambda$, so

$$
s \leqslant 1-\frac{2 \rho^{2}}{m},
$$

and in the majority of cases (13) holds with equality.

For example consider the lattice $D_{4}$. The first shell consists of the 24 points given in (6), and corresponds to $m=1$. The points $(1 / \sqrt{2})(1,1,0,0)$ and $(1 / \sqrt{2})(1,0,1,0)$ are at a distance $2 r=1=2 \rho$, and for this spherical code

$$
s=1-\frac{2 r^{2}}{m}=\frac{1}{2} \text {. }
$$

Similarly (13) holds with equality for most of the shells. On the other hand (13) can be improved for the shells corresponding to $m=2,4,8,16, \cdots$. These spherical codes also contain just 24 points, which after rescaling are either (6) again or

$$
( \pm 1,0,0,0), \cdots,(0,0,0, \pm 1), \frac{1}{2}( \pm 1, \pm 1, \pm 1, \pm 1) .
$$

For these codes $s$ is still $1 / 2$, but $r$ is $\sqrt{m} / 2$.

\section{CODES With Bounded ENERGY}

A spherical code represents a set of signals for the Gaussian channel in which each signal has the same energy [50]. A signal set with bounded energy may be obtained by taking all the centers of a packing $\Lambda$ that are within or on a large sphere of radius $\rho_{0}$. The total number of such centers is

$$
S\left(\rho_{0}\right):=\sum_{m \leqslant \rho_{0}^{2}} N_{m}
$$

The values of $S\left(\rho_{0}\right)$ are not included in the Tables for two

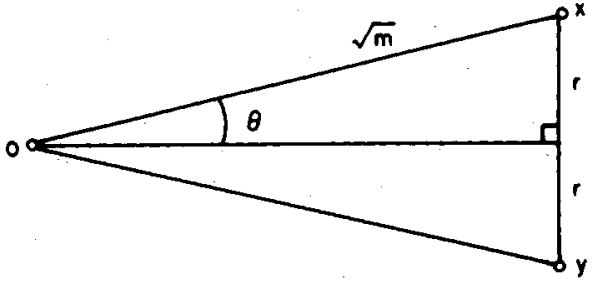

Fig. 1 The maximum dot product of the spherical code consisting of all points in $\Lambda$ at distance $\sqrt{m}$ from 0 is $\cos 2 \theta=1-2(r / \sqrt{m})^{2}$, where $2 r$ is the smallest distance between two such points.

reasons: 1) they are easily found by summing $N_{m}$; and 2) there is an excellent approximation to $S\left(\rho_{0}\right)$ given by

$$
S\left(\rho_{0}\right) \approx \Delta \cdot\left(\frac{\rho_{0}}{\rho}\right)^{n},
$$

or

$$
S\left(\rho_{0}\right) \approx \frac{V_{n} \rho_{0}^{n}}{\operatorname{det} \Lambda}
$$

if $\Lambda$ is a lattice packing. Also if $\Lambda$ corresponds to an integral quadratic form (see Section XI) then a theorem of Val'fiš [61] states that

$$
S\left(\rho_{0}\right)=\frac{V_{n} \rho_{0}^{n}}{\operatorname{det} \Lambda}+O\left(\rho_{0}^{n-1}\right),
$$

and so the error in the approximations (16) or (17) is of smaller order than the main term. Furthermore (18) gives a crude estimate for the order of magnitude of $N_{m}$. Since

$$
N_{m}=S(\sqrt{m})-S(\sqrt{m-1}),
$$

(18) implies

$$
c_{1} m^{(n / 2)-1}<N_{m}<c_{2} m^{(n / 2)-1}
$$

for some positive constants $c_{1}, c_{2}$.

\section{Connections with Number Theory}

A very old problem asks for the number of ways of expressing an integer $m$ as the sum of four squares, or in other words for the number of quadruples of integers $\left(u_{1}, u_{2}, u_{3}, u_{4}\right)$ such that

$$
u_{1}^{2}+u_{2}^{2}+u_{3}^{2}+u_{4}^{2}=m .
$$

For example when $m$ is 2 there are 24 solutions, given (ignoring the factor $\sqrt{2}$ ) by (6): we agree to count $(1,1,0,0)$, $(1,0,1,0)$, etc., as different solutions. A moment's thought shows that the general answer is given by the coefficient of $q^{m}$ in the expansion of

$$
\left(1+2 q+2 q^{4}+2 q^{9}+\cdots\right)^{4}=\theta_{3}(z)^{4}
$$

in powers of $q$. However, this power series is also the theta function of the lattice $Z^{4}$ of integer points in $R^{4}$ :

$$
\theta_{3}(z)^{4}=\Theta_{Z^{4}}(z) \text {. }
$$

In other words the coefficient of $q^{m}$ in this theta function gives the number of solutions to (20). Call this number $r_{4}(m)$. There is in fact a simple formula for this number, 
due to Jacobi:

$$
r_{4}(m)= \begin{cases}8 \sum_{d \mid m} d, & \text { if } m \text { is odd } \\ 24 \sum_{d \mid m, d \text { odd }} d, & \text { if } m \text { is even. }\end{cases}
$$

(See [28, thm. 386], [45, sec. 83], [46, eq. (7.4.23)].)

To generalize this, suppose $\Lambda$ is a lattice of dimension $n$ in $\boldsymbol{R}^{n}$ with the property that $\|\boldsymbol{x}\|$ is always an integer for $x \in \Lambda$. The integral quadratic form associated with $\Lambda$ is

$$
f\left(u_{1}, \cdots, u_{n}\right):=\sum_{i, j, k=1}^{n} u_{i} M_{i j} M_{k j} u_{k}
$$

or in vector notation

$$
f(\boldsymbol{u})=\boldsymbol{u} M M^{\mathrm{tr}} \boldsymbol{u}^{\mathrm{tr}}, \quad \text { for } \boldsymbol{u} \in Z^{n} .
$$

As $\boldsymbol{u}$ runs through $\boldsymbol{Z}^{n}, \boldsymbol{x}=\boldsymbol{u} M$ runs through $\Lambda$, and

$$
f(\boldsymbol{u})=\boldsymbol{x} \boldsymbol{x}^{\mathrm{l}}=\|\boldsymbol{x}\| \text {. }
$$

Thus the theta function of $\Lambda$ can be rewritten as

$$
\begin{aligned}
\Theta_{\Lambda}(z) & =\sum_{u \in Z^{n}} q^{u M M^{\mathrm{tr}} u^{\mathrm{tr}}} \\
& =\sum_{m=0}^{\infty} N_{m} q^{m},
\end{aligned}
$$

and the coefficient $N_{m}$ is equal to the number of solutions to the diophantine equation

$$
\boldsymbol{u} M M^{\mathrm{tr}} \boldsymbol{u}^{\mathrm{tr}}=m .
$$

In the above example $M$ is the $4 \times 4$ identity matrix $I_{4}$ and the quadratic form is $u_{1}^{2}+u_{2}^{2}+u_{3}^{2}+u_{4}^{2}$.

This link with number theory makes it possible to apply the vast literature on diophantine equations and modular forms to the study of lattices [17], [20], [24]-[28], [30], [37], [45], [46], [59], [61].

\section{DESCRIPTION OF SOME IMPORTANT LatTICES}

In the following sections we describe some of the most important lattices and give tables of their theta functions. In view of the construction in Section IX these are also tables of spherical codes. The maximum dot product of any of these codes is given by (12) and (13). For lattices in higher dimensions see [33], [34], [48], [51], [52].

Our notation is that

$\begin{array}{ll}d & \text { dimension of } \Lambda, \\ M & \text { generator matrix, } \\ \rho & \text { radius, } \\ \operatorname{det} & \text { determinant, } \\ \tau & \text { kissing number, } \\ \Delta & \text { density, } \\ \Theta_{\Lambda}(z) & \text { theta function, } \\ N_{m} & \text { number of centers } \boldsymbol{x} \in \Lambda \text { with }\|\boldsymbol{x}\|=m .\end{array}$

\section{The CUbic LatTice $\boldsymbol{Z}^{n}$ IN $\boldsymbol{R}^{n}$}

The simplest lattice is $Z^{n}$, which consists of all points in $\boldsymbol{R}^{n}$ with integer coordinates. For this lattice $d=n, M=I_{n}$,

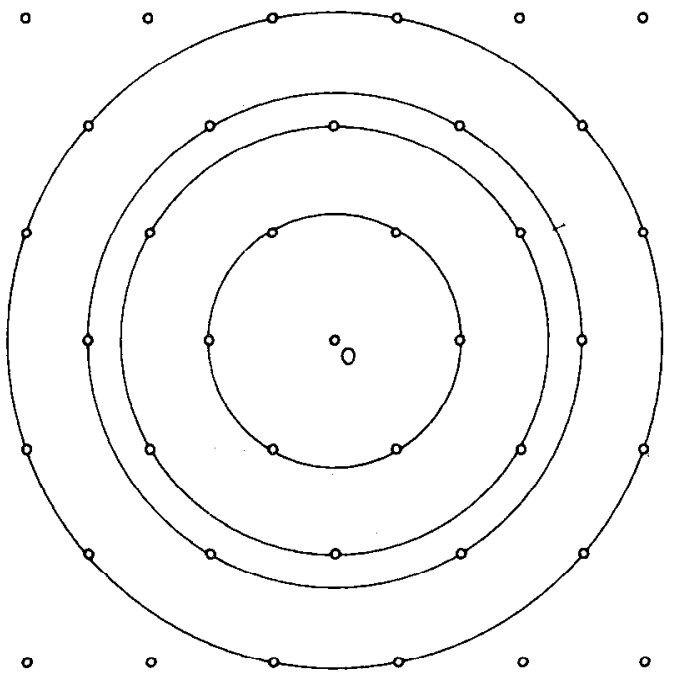

Fig. 2 The hexagonal lattice $A_{2}$ in the plane. The first five shells around the origin contain $1,6,6,6$, and 12 points, respectively.

$$
\begin{aligned}
\rho=1 / 2, \operatorname{det}=1, \tau=2 n, \Delta & =V_{n} / 2^{n}, \text { and } \\
\Theta_{Z^{n}}(z) & =\theta_{3}(z)^{n} \\
& =\sum_{m=0}^{\infty} r_{n}(m) q^{m} .
\end{aligned}
$$

The coefficient $r_{n}(m)$ is the number of ways of representing $m$ as a sum of $n$ squares. There are explicit formulas for small even values of $m$. For example

$r_{2}(m)=4|\{d \mid n, d \equiv 1(\bmod 4)\}|$

$$
-4|\{d \mid n, d \equiv 3(\bmod 4)\}|
$$

$r_{4}(m)$ is given by $(21)$;

$$
r_{6}(m)=16 \sum_{d \mid m} \chi\left(d^{\prime}\right) d^{2}-4 \sum_{d \mid m} \chi(d) d^{2},
$$

where $d d^{\prime}=m$ and $\chi(d)$ is $1,-1$, or 0 according as $d$ is of the form $4 k+1,4 k-1$, or $2 k$; and

$$
r_{8}(m)=16(-1)^{m} \sum_{d \mid m}(-1)^{d} d^{3} .
$$

Much more is known about $r_{n}(m)$ - sec the references given at the end of Section XI.

\section{The Two-Dimensional Lattice $A_{2}$}

This familiar lattice is shown in Fig. 2, and is spanned by the vectors $(1,0)$ and $(-1 / 2, \sqrt{3} / 2)$. Thus $d=2$,

$$
M=\left(\begin{array}{cc}
1 & 0 \\
-\frac{1}{2} & \frac{\sqrt{3}}{2}
\end{array}\right),
$$

$\rho=1 / 2, \operatorname{det}=\sqrt{3} / 2, \tau=6$, and the density is

$$
\Delta=\frac{\pi}{2 \sqrt{3}}=0.9069 \cdots,
$$

which is the highest attainable in $\boldsymbol{R}^{2}$ (see [47, p. 11]). The 
associated quadratic form is

$$
u^{2}-u v+v^{2}
$$

and the theta function is

$$
\begin{aligned}
\Theta_{A_{2}}(z)= & \sum_{u, v=-\infty}^{\infty} q^{u^{2}-u v+v^{2}} \\
= & \sum_{u, v=-\infty}^{\infty} q^{(u-(1 / 2) v)^{2}+(3 / 4) v^{2}} \\
= & \sum_{\substack{u, v=-\infty \\
v \text { even }}}^{\infty} q^{(u-(1 / 2) v)^{2}+(3 / 4) v^{2}} \\
& +\sum_{\substack{u, v=-\infty \\
v \text { odd }}}^{\infty} q^{(u-(1 / 2) v)^{2}+(3 / 4) v^{2}} \\
= & \sum_{r, s=-\infty}^{\infty} q^{r^{2}+3 s^{2}+} \sum_{r, s=-\infty}^{\infty} q^{(r+(1 / 2))^{2}+3(s+(1 / 2))^{2}} \\
= & \theta_{3}(z) \theta_{3}(3 z)+\theta_{2}(z) \theta_{2}(3 z) \\
= & \phi_{0}(z) \text { (say). }
\end{aligned}
$$

If we write

$$
\Theta_{A_{2}}(z)=\sum_{m=0}^{\infty} N_{m} q^{m},
$$

then $N_{m}$ is the number of times (29) represents $m$. It follows from the standard theory, ${ }^{3}$ writing

$$
N^{\prime}(m)=\frac{1}{6} N_{m},
$$

that $N^{\prime}(m)$ is multiplicative, i.e., satisfies

$N^{\prime}(r s)=N^{\prime}(r) N^{\prime}(s)$,

$$
\text { whenever } r \text { and } s \text { are relatively prime }
$$

(see [28]). Therefore it is sufficient to calculate $N^{\prime}(m)$ when $m=p^{a}$ is a power of a prime. These values are

$$
\begin{aligned}
& N^{\prime}\left(3^{a}\right)=1, \quad \text { for all } a, \\
& N^{\prime}\left(p^{a}\right)=a+1, \quad \text { if } p \equiv 1(\bmod 3), \\
& N^{\prime}\left(p^{a}\right)= \begin{cases}0, & \text { if } p \equiv 2(\bmod 3), \quad a \text { odd, } \\
1, & \text { if } \equiv 2(\bmod 3), \quad a \text { even. }\end{cases}
\end{aligned}
$$

The theta function begins

$$
\Theta_{A_{2}}(z)=1+6 q+6 q^{3}+6 q^{4}+12 q^{7}+\cdots
$$

(see Fig. 2), and further values are given in Table I.

\section{The $n$-Dimensional Lattice $A_{n}$}

$A_{2}$ may be generalized to higher dimensions as follows. We describe $A_{n}$ as an $n$-dimensional lattice in $\boldsymbol{R}^{n+1}$. Thus for $n \geqslant 2$ let

$$
A_{n}:=\left\{x \in Z^{n+1}\left|\sum_{j=1}^{n+1}\right| x_{j}=0\right\} .
$$

\footnotetext{
${ }^{3}$ Since (21) has class number 1 .
}

TABLE I

THE HEXAGONAL LATTICE $A_{2}$ IN $R^{2}$
\begin{tabular}{||c|c|c|c||c|c||}
\hline \hline$m$ & $\frac{1}{6} N_{m}$ & $m$ & $\frac{1}{6} N_{m}$ & $m$ & $\frac{1}{6} N_{m}$ \\
\hline & & & & & \\
0 & $1 / 6$ & 64 & 1 & 147 & 3 \\
1 & 1 & 67 & 2 & 148 & 2 \\
3 & 1 & 73 & 2 & 151 & 2 \\
4 & 1 & 75 & 1 & 156 & 2 \\
7 & 2 & 76 & 2 & 157 & 2 \\
9 & 1 & 79 & 2 & 163 & 2 \\
12 & 1 & 81 & 1 & 169 & 3 \\
13 & 2 & 84 & 2 & 171 & 2 \\
16 & 1 & 91 & 4 & 172 & 2 \\
19 & 2 & 93 & 2 & 175 & 2 \\
21 & 2 & 97 & 2 & 181 & 2 \\
25 & 1 & 100 & 1 & 183 & 2 \\
27 & 1 & 103 & 2 & 189 & 2 \\
28 & 2 & 108 & 1 & 192 & 1 \\
31 & 2 & 109 & 2 & 193 & 2 \\
36 & 1 & 111 & 2 & 196 & 3 \\
37 & 2 & 112 & 2 & 199 & 2 \\
39 & 2 & 117 & 2 & 201 & 2 \\
43 & 2 & 121 & 1 & 208 & 2 \\
48 & 1 & 124 & 2 & 211 & 2 \\
49 & 3 & 127 & 2 & 217 & 4 \\
52 & 2 & 129 & 2 & 219 & 2 \\
57 & 2 & 133 & 4 & 223 & 2 \\
61 & 2 & 139 & 2 & 225 & 1 \\
63 & 2 & 144 & 1 & 228 & 2 \\
\hline
\end{tabular}

This definition of $A_{2}$ agrees (apart from a rotation) with the definition in the previous section if the old version is rescaled by multiplying it by $c=\sqrt{2}$. Then $A_{n}$ has $d=n$,

$$
M=\left[\begin{array}{rrrrr}
1 & -1 & 0 & \cdots & 0 \\
1 & 0 & -1 & \cdots & 0 \\
1 & 0 & 0 & & -1
\end{array}\right],
$$

an $n \times(n+1)$ matrix, $\rho=1 / \sqrt{2}$, det $=\sqrt{n+1}$, and $\tau=$ $2 n(n-1)$. We may regard $A_{n}$ as a lattice in $\boldsymbol{R}^{n}$ by restricting our attention to the hyperplane

$$
\left\{\boldsymbol{x} \in \boldsymbol{R}^{n+1} \mid x_{1}+\cdots+x_{n+1}=0\right\} .
$$

Looked at in this way, $A_{n}$ has density

$$
\Delta=\frac{V_{n}}{2^{n / 2} \sqrt{n+1}}
$$

The theta function of $A_{n}$ is most simply expressed in terms of the more general Jacobi theta function [62, ch. XXI]

$$
\theta_{3}(\xi \mid z):=\sum_{m=-\infty}^{\infty} e^{2 m i \xi+\pi i z m^{2}}
$$

$\theta_{2}(z), \theta_{3}(z)$, and $\theta_{4}(z)$ may be expressed in terms of this function by

$$
\begin{aligned}
& \theta_{2}(z)=e^{\pi i z / 4} \theta_{3}\left(\frac{\pi z}{2} \mid z\right), \\
& \theta_{3}(z)=\theta_{3}(0 \mid z), \\
& \theta_{4}(z)=\theta_{3}\left(\frac{\pi}{2} \mid z\right) .
\end{aligned}
$$

Then the theta function of $A_{n-1}$ is

$$
\Theta_{A_{n-1}}(z)=\frac{1}{n \theta_{3}(n z)} \sum_{k=0}^{n-1} \theta_{3}\left(\frac{k \pi}{n} \mid z\right)^{n} .
$$

To prove this, observe that $A_{n-1}$ is a subgroup of the 
lattice

$$
L_{n}:=\left\{\boldsymbol{x} \in \boldsymbol{Z}^{n}\left|\sum_{j=1}^{n}\right| x_{j} \equiv 0(\bmod n)\right\},
$$

and in fact we may write

$$
L_{n}=\bigcup_{m=-\infty}^{\infty}\left\{(m, m, \cdots, m)+A_{n-1}\right\} .
$$

Therefore

$$
\begin{aligned}
\Theta_{L_{n}}(z) & =\sum_{m=-\infty}^{\infty} q^{n m^{2} \Theta_{A_{n-1}}(z)} \\
& =\theta_{3}(n z) \Theta_{A_{n-1}}(z) .
\end{aligned}
$$

To find the theta function of $L_{n}$ we weight each point $x$ of $Z^{n}$ according to the value of $\Sigma x_{j}$. Let

$$
\chi(\boldsymbol{x}):=e^{2 \pi i \Sigma_{j=1}^{n} x_{j} / n}, \quad \text { for } \boldsymbol{x} \in \boldsymbol{R}^{n},
$$

and

$$
\Theta_{\chi^{k}, Z^{n}}(z):=\sum_{x \in Z^{n}} \chi^{k}(x) q^{x \cdot x}
$$

for $k=0,1, \cdots, n-1$. Then

$$
\begin{aligned}
\Theta_{x^{k}, Z^{n}}(z) & =\left(\sum_{x \in Z} \chi^{k}(x) q^{x^{2}}\right)^{n} \\
& =\theta_{3}\left(\frac{k \pi}{n} \mid z\right)^{n}
\end{aligned}
$$

from (33). Finally the sum of

$$
\Theta_{\chi^{k}, Z^{n}}(z)
$$

over $k=0,1, \cdots, n-1$ picks out those $\boldsymbol{x} \in \boldsymbol{Z}^{n}$ with $\Sigma x_{j} \equiv 0$ $(\bmod n)$ :

$$
\Theta_{L_{n}}(z)=\frac{1}{n} \sum_{k=0}^{n-1} \Theta_{\chi^{k}, Z^{n}}(z),
$$

and (34) follows from (35), (38), (39).

\section{The $n$-Dimensional Lattice $D_{n}$}

The lattice packing $D_{n}$ in $\boldsymbol{R}^{n}$ is obtained by applying Construction $\mathrm{A}$ to the code consisting of all binary vectors of even weight, ${ }^{4}$ and then rescaling by multiplying all the centers by $1 / \sqrt{2}$. Alternatively, color the points of $\boldsymbol{Z}^{n}$ red and blue with a checkerboard coloring, take the red points, and multiply by $1 / \sqrt{2}$. For this lattice $d=n$,

$$
\begin{aligned}
M & =\frac{1}{\sqrt{2}}\left[\begin{array}{ccccc}
2 & 0 & 0 & \cdots & 0 \\
1 & 1 & 0 & \cdots & 0 \\
1 & 0 & 1 & \cdots & 0 \\
1 & 0 & 0 & \cdots & 1
\end{array}\right], \\
\rho & =\frac{1}{2} \\
\operatorname{det} & =2^{-(n-2) / 2} \\
\tau & =2 n(n-1)
\end{aligned}
$$

${ }^{4}$ Construction A: if $C$ is a binary code of length $n$, the set of centers $c+2 x\left(c \in C, x \in Z^{n}\right)$ forms a sphere packing in $R^{n}$. Most of the properties of this packing can be obtained directly from the code $C$ - - see [52] for details. and

$$
\Delta=V_{n} 2^{-(n+2) / 2} .
$$

For $n=3,4$, and $5 D_{n}$ is the densest possible lattice packing (as well as the densest known packing, although for $n=3$ and 5 there are equally dense nonlattice packings [34]). Also

$$
\begin{aligned}
\Theta_{D_{n}}(z) & =\frac{1}{2}\left\{\theta_{3}\left(\frac{z}{2}\right)^{n}+\theta_{4}\left(\frac{z}{2}\right)^{n}\right\} \\
& =\sum_{m=0}^{\infty} r_{n}(2 m) q^{m},
\end{aligned}
$$

using the notation of (25). This begins

$$
\Theta_{D_{n}}(z)=1+2 n(n-1) q+2 n q^{2}+2 n(n-1) q^{4}+\cdots .
$$

The dual lattice $D_{n}^{\perp}(n \geqslant 3)$ can of course be obtained by finding the dual of $D_{n}$, or more simply by applying Construction $A$ to the repetition code $\{(0,0, \cdots, 0),(1,1, \cdots, 1)\}$. For this lattice $d=n$,

$$
\begin{aligned}
M & =\left[\begin{array}{llllll}
2 & 0 & 0 & \cdots & 0 & 0 \\
0 & 2 & 0 & \cdots & 0 & 0 \\
0 & 0 & 2 & \cdots & 0 & 0 \\
0 & 0 & 0 & \cdots & 2 & 0 \\
1 & 1 & 1 & \cdots & 1 & 1
\end{array}\right], \\
\rho & =\left\{\begin{array}{lll}
\sqrt{3} / 2, & \text { if } n-3, \\
1, & \text { if } n \geqslant 4,
\end{array}\right. \\
\operatorname{det} & =2^{n-1}, \\
\tau & = \begin{cases}8, & \text { if } n=3, \\
24, & \text { if } n=4, \\
2 n, & \text { if } n \geqslant 5,\end{cases} \\
\Delta & =\left\{\begin{array}{l}
\frac{\pi \sqrt{3}}{8}=0.680175 \cdots, \\
\frac{V_{n}}{2^{n-1}},
\end{array}\right.
\end{aligned}
$$

and

$$
\Theta_{D_{n}^{\perp}}(z)=\theta_{3}(4 z)^{n}+\theta_{2}(4 z)^{n}
$$

The case $n-3$. References for these three-dimensional packings are [1, ch. V], [14, ch. IV] and [15, ch. 9]. $D_{3}$ is the familiar face-centered cubic lattice (as seen in a square or triangular pyramid of billiard balls), and has density $\Delta=$ $\pi / 3 \sqrt{2}=0.740480 \cdots$. There is a complicated formula for the coefficients $r_{3}(2 \mathrm{~m})$ (see [17, vol. II, chapter VII]). The dual lattice $D_{3}{ }^{+}$is the body-centered cubic lattice. The coefficients of the theta functions are given in Tables II and III.

There is a further packing in $\boldsymbol{R}^{3}$ that has not yet been mentioned. This is the hexagonal close-packing, which is a nonlattice packing with the same density and kissing number as $D_{3}$. It may be defined to be the union of the lattice $L$ spanned by $(\sqrt{3}, 0,0),(\sqrt{3} / 2,3 / 2,0)$, and $(0,0,2 \sqrt{2})$, and 
TABLE II

The Face-Centered Cubic Lattice $D_{3}$ in $\boldsymbol{R}^{3}$ (The Table Gives $\frac{1}{6} N_{m}$ FOR $\left.m=10 r+s\right)$

\begin{tabular}{||c|cccccccccc||}
\hline$r / s$ & 0 & 1 & 2 & 3 & 4 & 5 & 6 & 7 & 8 & 9 \\
\hline 0 & $1 / 6$ & 2 & 1 & 4 & 2 & 4 & $4 / 3$ & 8 & 1 & 6 \\
1 & 4 & 4 & 4 & 12 & 0 & 8 & 2 & 8 & 5 & 12 \\
2 & 4 & 8 & 4 & 8 & $4 / 3$ & 14 & 4 & 16 & 8 & 4 \\
3 & 0 & 16 & 1 & 16 & 8 & 8 & 6 & 20 & 4 & 8 \\
4 & 4 & 8 & 8 & 20 & 4 & 20 & 0 & 16 & 4 & 18 \\
5 & 5 & 8 & 12 & 12 & $16 / 3$ & 24 & 0 & 16 & 12 & 12 \\
6 & 8 & 20 & 0 & 24 & 2 & 8 & 8 & 28 & 8 & 16 \\
7 & 8 & 8 & 5 & 32 & 4 & 20 & 12 & 16 & 0 & 16 \\
8 & 4 & 18 & 16 & 20 & 8 & 24 & 4 & 24 & 4 & 16 \\
9 & 12 & 24 & 8 & 24 & 0 & 8 & $4 / 3$ & 40 & 9 & 20 \\
\hline
\end{tabular}

TABLE III

The Body-Centered Cubic LatTice $D_{3}^{\perp}$ IN $R^{3}$

\begin{tabular}{|r|r||r|r||r|r||}
\hline$m$ & \multicolumn{1}{|c||}{$N_{m}$} & \multicolumn{1}{|c|}{$m$} & \multicolumn{1}{|c||}{$N_{m}$} & $m$ & \multicolumn{1}{|c|}{$N_{m}$} \\
& & & & & \\
3 & 1 & 67 & 24 & 136 & 48 \\
3 & 8 & 68 & 48 & 139 & 72 \\
4 & 6 & 72 & 36 & 140 & 48 \\
8 & 12 & 75 & 56 & 144 & 30 \\
11 & 24 & 76 & 24 & 147 & 56 \\
12 & 8 & 80 & 24 & 148 & 24 \\
16 & 6 & 83 & 72 & 152 & 72 \\
19 & 24 & 84 & 48 & 155 & 96 \\
20 & 24 & 88 & 24 & 160 & 24 \\
24 & 24 & 91 & 48 & 163 & 24 \\
27 & 32 & 96 & 24 & 164 & 96 \\
32 & 12 & 99 & 72 & 168 & 48 \\
35 & 48 & 100 & 30 & 171 & 120 \\
36 & 30 & 104 & 72 & 172 & 24 \\
40 & 24 & 107 & 72 & 176 & 24 \\
43 & 24 & 108 & 32 & 179 & 120 \\
44 & 24 & 115 & 48 & 180 & 72 \\
48 & 8 & 116 & 72 & 184 & 48 \\
51 & 48 & 120 & 48 & 187 & 48 \\
52 & 24 & 123 & 48 & 192 & 8 \\
56 & 48 & 128 & 12 & 195 & 96 \\
59 & 72 & 131 & 120 & 196 & 54 \\
64 & 6 & 132 & 48 & 200 & 84 \\
\hline
\end{tabular}

the coset

$$
L^{\prime}=\left(\frac{\sqrt{3}}{2}, \frac{1}{2}, \sqrt{2}\right)+L .
$$

By following the same kind of algebra used to derive (30), one can show that the theta functions of $L$ and $L^{\prime}$ are respectively

$$
\theta_{3}(8 z) \phi_{0}(3 z)
$$

and

$$
\frac{1}{2} \theta_{2}(8 z)\left\{\phi_{0}(z)-\phi_{0}(3 z)\right\}
$$

Therefore the theta function of the hexagonal close-packing is

$$
\begin{aligned}
\phi_{0}(3 z)\left\{\theta_{3}(8 z)-\frac{1}{2} \theta_{2}(8 z)\right\} & +\frac{1}{2} \phi_{0}(z) \theta_{2}(8 z) \\
& =1+12 q^{3}+6 q^{6}+2 q^{8}+\cdots
\end{aligned}
$$

- see Table IV.

The case $n=4$ (see also Sec. V). $D_{4}$ is a self-dual lattice: $D_{4}=D_{4}^{\perp}$. The coefficients $N_{m}=r_{4}(2 m)$ of the theta function are given by the second formula in (21), and the first 50 terms are given in Table V. Furthermore $(24)^{-1} N_{m}$ is multiplicative. It is worth mentioning that $N_{m}$ is the number of integral quaternions of norm $m[15$, p. 25$]$.
TABLE IV

The Hexagonal Close-Packing: A Nonlattice Packing in $\boldsymbol{R}^{3}$ ('IHE 'TABLE Gives $\frac{1}{6} N_{m}$ FOR $m=10 r+s$ )

\begin{tabular}{||c|cccccccccc||}
\hline$r / s$ & 0 & 1 & 2 & 3 & 4 & 5 & 6 & 7 & 8 & 9 \\
\hline 0 & $1 / 6$ & 0 & 0 & 2 & 0 & 0 & 1 & 0 & $1 / 3$ & 3 \\
1 & 0 & 2 & 1 & 0 & 0 & 2 & 0 & 2 & 1 & 1 \\
2 & 2 & 1 & 1 & 0 & 0 & 2 & 0 & 2 & 0 & 4 \\
3 & 2 & 2 & $1 / 3$ & 2 & 1 & 4 & 1 & 2 & 0 & 4 \\
4 & 0 & 2 & 0 & 1 & 4 & 2 & 2 & 4 & 1 & 2 \\
5 & 0 & 4 & 0 & 4 & 3 & 2 & 2 & 4 & 0 & 2 \\
6 & 0 & 2 & 0 & 6 & 0 & 4 & 2 & 3 & 2 & 4 \\
7 & 2 & 8 & $1 / 3$ & 0 & 0 & 6 & 0 & 0 & 4 & 2 \\
8 & 2 & 7 & 1 & 2 & 4 & 2 & 0 & 2 & 0 & 6 \\
9 & 0 & 2 & 4 & 12 & 2 & 4 & 0 & 2 & 0 & 8 \\
& & & & & & & & & & \\
\hline
\end{tabular}

TABLE V The LATTICE $D_{4}$ IN $\boldsymbol{R}^{4}$

\begin{tabular}{||c|c||c|c||}
\hline \hline$m$ & $(24)^{-1} N_{m}$ & $m$ & $(24)^{-1} N_{m}$ \\
1 & 1 & 26 & 14 \\
2 & 1 & 27 & 40 \\
3 & 4 & 28 & 8 \\
4 & 1 & 29 & 30 \\
5 & 6 & 30 & 24 \\
6 & 4 & 31 & 32 \\
7 & 8 & 32 & 1 \\
8 & 1 & 33 & 48 \\
9 & 13 & 34 & 18 \\
10 & 6 & 35 & 48 \\
11 & 12 & 36 & 13 \\
12 & 4 & 37 & 38 \\
13 & 14 & 38 & 20 \\
14 & 8 & 39 & 56 \\
15 & 24 & 40 & 6 \\
16 & 1 & 41 & 42 \\
17 & 18 & 42 & 32 \\
18 & 13 & 43 & 44 \\
19 & 20 & 44 & 12 \\
20 & 6 & 45 & 78 \\
21 & 32 & 46 & 24 \\
22 & 12 & 47 & 48 \\
23 & 24 & 48 & 4 \\
24 & 4 & 49 & 57 \\
25 & 31 & 50 & 31 \\
\hline
\end{tabular}

XVII. The Lattices $E_{6}, E_{7}, E_{8}$

The lattice packings $E_{6}$ in $\boldsymbol{R}^{6}, E_{7}$ in $\boldsymbol{R}^{7}$, and $E_{8}$ in $\boldsymbol{R}^{8}$ are the densest possible lattice packings in these dimensions (and also the densest known packings there). $E_{6}$ is most easily constructed from the ternary code $\{000,111,222\}$. The complex version of Construction $\mathrm{A}$ then produces a complex lattice ${ }^{5}$ in $C^{3}$ with generator matrix

$$
\frac{1}{\sqrt{3}}\left(\begin{array}{ccc}
i \sqrt{3} & 0 & 0 \\
0 & i \sqrt{3} & 0 \\
1 & 1 & 1
\end{array}\right)
$$

- see [54, sec. 5.8.2]. By regarding this as a real lattice in $\boldsymbol{R}^{6}$ we obtain $E_{6}$, for which $d=6$,

$$
M=\frac{1}{\sqrt{3}}\left[\begin{array}{cccccc}
0 & 0 & 0 & \sqrt{3} & 0 & 0 \\
0 & 0 & 0 & 0 & \sqrt{3} & 0 \\
1 & 1 & 1 & 0 & 0 & 0 \\
\frac{3}{2} & 0 & 0 & \frac{\sqrt{3}}{2} & 0 & 0 \\
0 & \frac{3}{2} & 0 & 0 & \frac{\sqrt{3}}{2} & 0 \\
\frac{1}{2} & \frac{1}{2} & \frac{1}{2} & \frac{-\sqrt{3}}{2} & \frac{-\sqrt{3}}{2} & \frac{-\sqrt{3}}{2}
\end{array}\right]
$$

${ }^{5}$ Strictly speaking, this is a $Z\left[e^{2 \pi i / 3}\right]$-module in $C^{3}$ 
$\rho=1 / 2, \operatorname{det}=\sqrt{3} / 2^{3}, \tau=72, \Delta=\pi^{3} / 48 \sqrt{3}=0.372948 \cdots$ and

$$
\begin{aligned}
\Theta_{E_{6}}(z) & =\phi_{0}(z)^{3}+\frac{1}{4}\left\{\phi_{0}\left(\frac{z}{3}\right)-\phi_{0}(z)\right\}^{3} \\
& =1+72 q+270 q^{2}+\cdots .
\end{aligned}
$$

$E_{7}$ may be obtained by applying Construction $\mathrm{A}$ to the little Hamming code of length seven containing eight codewords. For this lattice $d=7$,

$$
M=\frac{1}{2}\left[\begin{array}{lllllll}
2 & 0 & 0 & 0 & 0 & 0 & 0 \\
0 & 2 & 0 & 0 & 0 & 0 & 0 \\
0 & 0 & 2 & 0 & 0 & 0 & 0 \\
0 & 0 & 0 & 2 & 0 & 0 & 0 \\
1 & 1 & 1 & 0 & 1 & 0 & 0 \\
0 & 1 & 1 & 1 & 0 & 1 & 0 \\
0 & 0 & 1 & 1 & 1 & 0 & 1
\end{array}\right]
$$

$\rho=1 / 2, \quad \operatorname{det}=1 / 8, \quad \tau=126, \Delta=\pi^{3} / 105=0.295298 \cdots$, and the theta function is, from [52, thm. 6],

$$
\begin{aligned}
\Theta_{E_{7}}(z) & =\theta_{3}(z)^{7}+7 \theta_{3}(z)^{3} \theta_{2}(z)^{4} \\
& =1+126 q+756 q^{2}+\cdots
\end{aligned}
$$

$E_{8}$ is obtained similarly from the extended Hamming code of length eight. For this lattice $d=8$,

$$
M=\frac{1}{2}\left[\begin{array}{llllllll}
2 & 0 & 0 & 0 & 0 & 0 & 0 & 0 \\
0 & 2 & 0 & 0 & 0 & 0 & 0 & 0 \\
0 & 0 & 2 & 0 & 0 & 0 & 0 & 0 \\
0 & 0 & 0 & 2 & 0 & 0 & 0 & 0 \\
1 & 1 & 1 & 0 & 1 & 0 & 0 & 0 \\
0 & 1 & 1 & 1 & 0 & 1 & 0 & 0 \\
0 & 0 & 1 & 1 & 1 & 0 & 1 & 0 \\
1 & 1 & 1 & 1 & 1 & 1 & 1 & 1
\end{array}\right],
$$

$\rho=1 / 2, \operatorname{det}=1 / 16, \tau=240, \Delta=\pi^{4} / 384=0.253670 \cdots$, and the theta function is (see for example [52, eqs. (34), (47), (48)])

$$
\begin{aligned}
\Theta_{E_{8}}(z) & =\theta_{3}(z)^{8}+14 \theta_{3}(z)^{4} \theta_{2}(z)^{4}+\theta_{2}(z)^{8} \\
& =\frac{1}{2}\left\{\theta_{2}\left(\frac{z}{2}\right)^{8}+\theta_{3}\left(\frac{z}{2}\right)^{8}+\theta_{4}\left(\frac{z}{2}\right)^{8}\right\} \\
& =1+\sum_{m=1}^{\infty} N_{m} q^{m},
\end{aligned}
$$

where

$$
N_{m}=240 \sigma_{3}(m)
$$

and

$$
\sigma_{r}(m)=\sum_{d \mid m} d^{r}
$$

The coefficients lie in the range

$$
240 m^{3}<N_{m}<240 \zeta(3) m^{3} \approx 288.5 m^{3} .
$$

Furthermore $N_{m}$ is equal to the number of integral Cayley numbers of norm $m[15, \mathrm{ch} .2]$. The first fifty terms of these three theta functions are given in Table VI.
TABLE VI

THE LATTICES $E_{6}, E_{7}$, AND $E_{8}$ IN $\boldsymbol{R}^{6}, \boldsymbol{R}^{7}$, AND $\boldsymbol{R}^{8}$

\begin{tabular}{|c|c|c|c||}
\hline$m$ & $N_{m}\left(E_{6}\right)$ & $N_{m}\left(E_{7}\right)$ & $(240)^{-1} \cdot N_{m}\left(E_{8}\right)$ \\
\hline & 72 & 126 & 1 \\
2 & 270 & 756 & 9 \\
3 & 720 & 2072 & 28 \\
4 & 936 & 4158 & 73 \\
5 & 2160 & 7560 & 126 \\
6 & 2214 & 11592 & 252 \\
7 & 3600 & 16704 & 344 \\
8 & 4590 & 24948 & 585 \\
9 & 6552 & 31878 & 757 \\
10 & 5184 & 39816 & 1134 \\
11 & 10800 & 55944 & 1332 \\
12 & 9360 & 66584 & 2044 \\
13 & 12240 & 76104 & 2198 \\
14 & 13500 & 99792 & 3096 \\
15 & 17712 & 116928 & 3528 \\
16 & 14760 & 133182 & 4681 \\
17 & 25920 & 160272 & 4914 \\
18 & 19710 & 177660 & 6813 \\
19 & 26064 & 205128 & 6860 \\
20 & 28080 & 249480 & 9198 \\
21 & 36000 & 265104 & 9632 \\
22 & 25920 & 281736 & 11988 \\
23 & 47520 & 350784 & 12168 \\
24 & 37638 & 382536 & 16380 \\
25 & 43272 & 390726 & 15751 \\
& & & \\
\hline 26 & 45900 & 470232 & 19782 \\
\hline 27 & 59040 & 505568 & 20440 \\
28 & 46800 & 532800 & 25112 \\
29 & 75600 & 615384 & 24390 \\
30 & 51840 & 640080 & 31752 \\
31 & 69264 & 701568 & 29792 \\
32 & 73710 & 799092 & 37449 \\
33 & 88560 & 809424 & 37296 \\
34 & 62208 & 853776 & 44226 \\
35 & 108000 & 1006992 & 43344 \\
36 & 85176 & 1051974 & 55261 \\
37 & 98640 & 1031688 & 50654 \\
38 & 97740 & 1195992 & 61740 \\
39 & 122400 & 1286208 & 61544 \\
40 & 88128 & 1313928 & 73710 \\
41 & 151200 & 1469664 & 68922 \\
42 & 110700 & 1474704 & 86688 \\
43 & 133200 & 1547784 & 79508 \\
44 & 140400 & 1797768 & 97236 \\
45 & 157680 & 1776600 & 95382 \\
46 & 114048 & 1809360 & 109512 \\
47 & 198720 & 2104704 & 103824 \\
48 & 147600 & 2130968 & 131068 \\
49 & 176472 & 2123982 & 117993 \\
50 & 162270 & 2382156 & 141759 \\
& & & \\
\hline & & & \\
\hline
\end{tabular}

\section{The 12-DIMENSIONAL LATTICE $K_{12}$}

This is the densest packing known in $\boldsymbol{R}^{12}$ and like $E_{6}$ is most easily constructed from a complex lattice. The starting point is the three-dimensional self-dual code $g_{6}$ of length six over $\mathrm{GF}(4)$ with generator matrix

$$
\left[\begin{array}{cccccc}
1 & \omega & \omega & 1 & 0 & 0 \\
\omega & 1 & \omega & 0 & 1 & 0 \\
\omega & \omega & 1 & 0 & 0 & 1
\end{array}\right],
$$

where $\omega \in \mathrm{GF}(4)$ is a primitive cube root of unity. By applying the complex version of Construction $A$ to this code [53], [54], we obtain a lattice in $C^{6}$ with generator matrix

$$
\frac{1}{2}\left[\begin{array}{llllll}
2 & 0 & 0 & 0 & 0 & 0 \\
0 & 2 & 0 & 0 & 0 & 0 \\
0 & 0 & 2 & 0 & 0 & 0 \\
1 & \omega & \omega & 1 & 0 & 0 \\
\omega & 1 & \omega & 0 & 1 & 0 \\
\omega & \omega & 1 & 0 & 0 & 1
\end{array}\right],
$$

where now $\omega=e^{2 \pi i / 3}$. Then $K_{12}$ is obtained by regarding this as a real lattice in $R^{12}$. For this lattice $d=12, M$ is 


\begin{tabular}{|ccc|ccc|ccc|ccc|}
\hline 4 & 0 & 0 & 0 & 0 & 0 & 0 & 0 & 0 & 0 & 0 & 0 \\
0 & 4 & 0 & 0 & 0 & 0 & 0 & 0 & 0 & 0 & 0 & 0 \\
0 & 0 & 4 & 0 & 0 & 0 & 0 & 0 & 0 & 0 & 0 & 0 \\
\hline 2 & -1 & -1 & 2 & 0 & 0 & 0 & $\sqrt{3}$ & $\sqrt{3}$ & 0 & 0 & 0 \\
-1 & 2 & -1 & 0 & 2 & 0 & $\sqrt{3}$ & 0 & $\sqrt{3}$ & 0 & 0 & 0 \\
-1 & -1 & 2 & 0 & 0 & 2 & $\sqrt{3}$ & $\sqrt{3}$ & 0 & 0 & 0 & 0 \\
\hline 2 & 0 & 0 & 0 & 0 & 0 & $2 \sqrt{3}$ & 0 & 0 & 0 & 0 & 0 \\
0 & 2 & 0 & 0 & 0 & 0 & 0 & $-2 \sqrt{3}$ & 0 & 0 & 0 & 0 \\
0 & 0 & 2 & 0 & 0 & 0 & 0 & 0 & $-2 \sqrt{3}$ & 0 & 0 & 0 \\
\hline 1 & 1 & 1 & 1 & 0 & 0 & $\sqrt{3}$ & $\sqrt{3}$ & $\sqrt{3}$ & $-\sqrt{3}$ & 0 & 0 \\
1 & 1 & 1 & 0 & 1 & 0 & $\sqrt{3}$ & $-\sqrt{3}$ & $\sqrt{3}$ & 0 & $-\sqrt{3}$ & 0 \\
1 & 1 & 1 & 0 & 0 & 1 & $\sqrt{3}$ & $\sqrt{3}$ & $-\sqrt{3}$ & 0 & 0 & $-\sqrt{3}$ \\
\hline
\end{tabular}

Fig. 3 Generator matrix for the 12-dimensional lattice packing $K_{12}$.

TABLE VII

$K_{12}$, THE BEST PACKING KNOWN IN
\begin{tabular}{||c|c||c|c||}
\hline$m$ & $N_{m}$ & $m$ & $N_{00}$ \\
\hline 0 & 1 & 19 & 48009024 \\
1 & 0 & 20 & 64049832 \\
1 & 756 & 21 & 70709184 \\
2 & 22 & 102958128 \\
3 & 4032 & 22 & 124782336 \\
4 & 20412 & 23 & 146 \\
5 & 60480 & 24 & 142254252 \\
6 & 13986 & 25 & 189423360 \\
7 & 326592 & 26 & 237588120 \\
8 & 652928 & 27 & 248250240 \\
9 & 1020096 & 28 & 344391264 \\
10 & 2000376 & 29 & 397510848 \\
11 & 3132864 & 30 & 433936440 \\
12 & 4445532 & 31 & 554879808 \\
13 & 7185024 & 32 & 671393772 \\
14 & 10747296 & 33 & 677557440 \\
15 & 13148352 & 34 & 908374824 \\
16 & 21003948 & 35 & 1018507392 \\
17 & 27506304 & 36 & 1079894844 \\
18 & 33724404 & & \\
\hline
\end{tabular}

shown in Fig. $3, \rho=1 / 2$, det $=3^{3} / 2^{12}, \tau=756$, and

$$
\Delta=\frac{\pi^{6}}{19440}=0.0494542 \cdots
$$

Furthermore from the weight enumerator of $g_{6}$ we can write down immediately that

$$
\begin{aligned}
\Theta_{K_{12}}(z) & =\phi_{0}(2 z)^{6}+45 \phi_{0}(2 z)^{2} \phi_{1}(2 z)^{4}+18 \phi_{1}(2 z)^{6} \\
& =1+756 q^{2}+4032 q^{3}+\cdots,
\end{aligned}
$$

where $\phi_{0}(z)$ is defined in (30) and

$$
\begin{aligned}
\phi_{1}(z) & =\theta_{2}(z) \theta_{3}(3 z)+\theta_{2}(3 z) \theta_{3}(z) \\
& =\frac{1}{2} \theta_{2}\left(\frac{z}{4}\right) \theta_{2}\left(\frac{3 z}{4}\right) \\
& =2 q^{1 / 4}\left(1+q^{1 / 2}+2 q^{3 / 2}+q^{2}+2 q^{3}+\cdots\right) .
\end{aligned}
$$

The first 36 terms of the theta function are given in Table VII.

\section{The 16-Dimensional Lattice $\Lambda_{16}$}

Only a brief description is given here of $\Lambda_{16}$, the densest known lattice in $\boldsymbol{R}^{16}$, which is obtained by applying Construction $B$ to the first-order Reed-Muller code of length 16 (see [34, sec. 3.4], [52, ex. 9]). For this lattice $d=16, M$ is shown in Fig. 4, $\rho=2^{-1 / 2}$, det $=2^{-4}, \tau=4320$, and

$$
\Delta=\frac{\pi^{8}}{8 ! 2^{4}}=0.0147082 \cdots \text {. }
$$

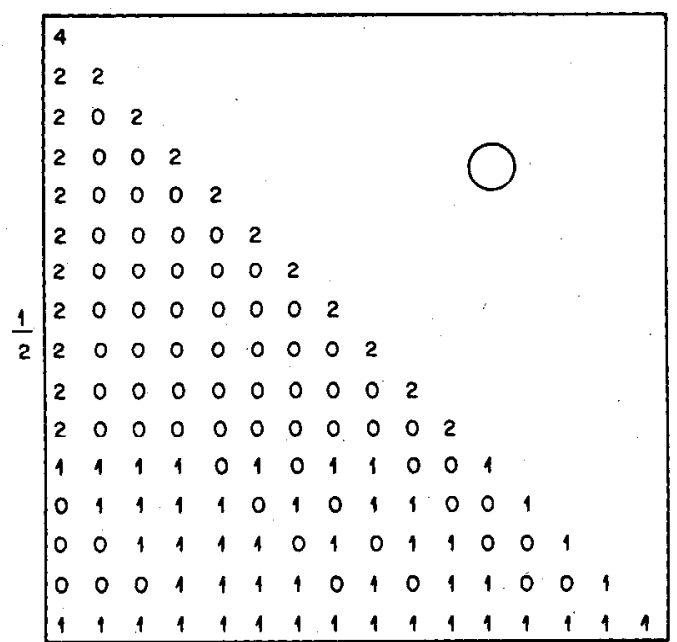

Fig. 4 Generator matrix for the 16-dimensional lattice packing $\Lambda_{16}$. The last six rows are a generator matrix for the first-order Reed-Muller code of length 16 .

TABLE VIII

$\Lambda_{16}$, THE BEST PACKING KNOWN IN $\boldsymbol{R}^{16}$
\begin{tabular}{||c|c||c|c||}
\hline$m$ & $N_{m}$ & $m$ & $N_{m}$ \\
\hline 0 & 1 & 16 & 85937797600 \\
1 & 0 & 17 & 11585617920 \\
2 & 4320 & 18 & 19590534240 \\
3 & 61440 & 19 & 25239859200 \\
4 & 522720 & 20 & 40979580480 \\
5 & 2211840 & 21 & 50877235200 \\
6 & 8960640 & 22 & 79783021440 \\
7 & 23224320 & 23 & 96134307840 \\
8 & 67154400 & 24 & 146902369920 \\
9 & 135168000 & 25 & 172337725440 \\
10 & 319809600 & 26 & 256900127040 \\
11 & 550195200 & 27 & 295487692800 \\
12 & 1147643520 & 28 & 431969276160 \\
13 & 1771683840 & 29 & 487058227200 \\
14 & 3371915520 & 30 & 699846624000 \\
15 & 4826603520 & & \\
\hline
\end{tabular}

Again the theta function can be written down from the weight enumerator of the code ([52, thm. 17]) and is

$$
\begin{aligned}
\Theta_{\Lambda_{16}}(z) & =\frac{1}{2}\left\{\theta_{2}(z)^{16}+\theta_{3}(z)^{16}+\theta_{4}(z)^{16}+30 \theta_{2}(z)^{8} \theta_{3}(z)^{8}\right\} \\
= & 1+4320 q^{2}+61440 q^{3}+\cdots .
\end{aligned}
$$

The first 30 terms are given in Table VIII.

At this point it is worth mentioning an interesting unsolved problem. The Nordstrom-Robinson code [36, ch. 2 , sec. 8], [42] is a union of the first-order Reed-Muller code above and seven of its translates; it is optimal in the sense that it contains the greatest number of codewords of any binary code of length 16 and minimum distance 6 . It is possible that there exists an analogous nonlattice packing, perhaps consisting of a union of $\Lambda_{16}$ and 15 translates. The theta function can be determined exactly and the first ten terms are given in Table IX. Such a packing would have an extremely high kissing number and density (see [52, open prob. 7]).

\section{The 24-Dimensional Leech Lattice $\Lambda_{24}$}

A great deal has already been written about this important lattice, the densest known packing in $\boldsymbol{R}^{24}$ [2], [4], 
TABLE IX

THE HyPOTHETICAL "NORDSTROM-ROBINSON" NONLATTICE PACKING IN $R^{16}$

\begin{tabular}{|c|c|}
\hline$m$ & $N_{m}$ \\
\hline & \\
0 & 1 \\
$1 / 2$ & 0 \\
1 & 0 \\
$3 / 2$ & 7680 \\
2 & 4320 \\
$5 / 2$ & 276480 \\
3 & 61440 \\
$7 / 2$ & 2903040 \\
4 & 522720 \\
$9 / 2$ & 16896000 \\
\hline
\end{tabular}

[9]-[12], [32], [34], [43], [52], [54], [55]. The centers are the points

$$
\frac{1}{2 \sqrt{2}}(0+2 c+4 x)
$$

and

$$
\frac{1}{2 \sqrt{2}}(1+2 c+4 y)
$$

where $\mathbf{0}=(0,0, \cdots, 0), \mathbf{1}=(1,1, \cdots, 1), \boldsymbol{c}$ is any codeword in the binary extended Golay code of length 24 , and $x \in Z^{24}$ and $y \in Z^{24}$ satisfy

$$
\begin{aligned}
& \sum_{i=1}^{24} x_{i} \equiv 0(\bmod 2) \\
& \sum_{i=1}^{24} y_{i} \equiv 1(\bmod 2)
\end{aligned}
$$

For this lattice $d=24, M$ is shown in Fig. 5, $\rho=1$, det $=1$, $\tau=196560$,

$$
\Delta=\frac{\pi^{12}}{12 !}=0.00192957 \cdots
$$

and

$$
\begin{aligned}
\Theta_{\Lambda_{\lambda 4}}(z) & =\Theta_{E_{8}}\left(z^{2}\right)^{3}-720 q^{2} \prod_{m=1}^{\infty}\left(1-q^{2 m}\right)^{24} \\
& =1+196560 q^{4}+16773120 q^{6}+\cdots .
\end{aligned}
$$

The first 40 terms are given in Table $\mathrm{X}$, together with their prime factors.

There is an explicit formula for $N_{m}$, the coefficient of $q^{m}:$

$$
N_{m}=\frac{65520}{691}\left(\sigma_{11}\left(\frac{m}{2}\right)-\tau\left(\frac{m}{2}\right)\right),
$$

where $\sigma_{11}(n)$ is defined by (47) and $\tau(n)$ is a Ramanujan number defined by

$$
q \prod_{n=1}^{\infty}\left(1-q^{n}\right)^{24}=\sum_{n=1}^{\infty} \tau(n) q^{n}
$$

Furthermore

$$
N_{m}=\frac{65520}{691} \sigma_{11}\left(\frac{m}{2}\right)+O\left(m^{6}\right),
$$

the second term on the right being of much smaller order than the first.

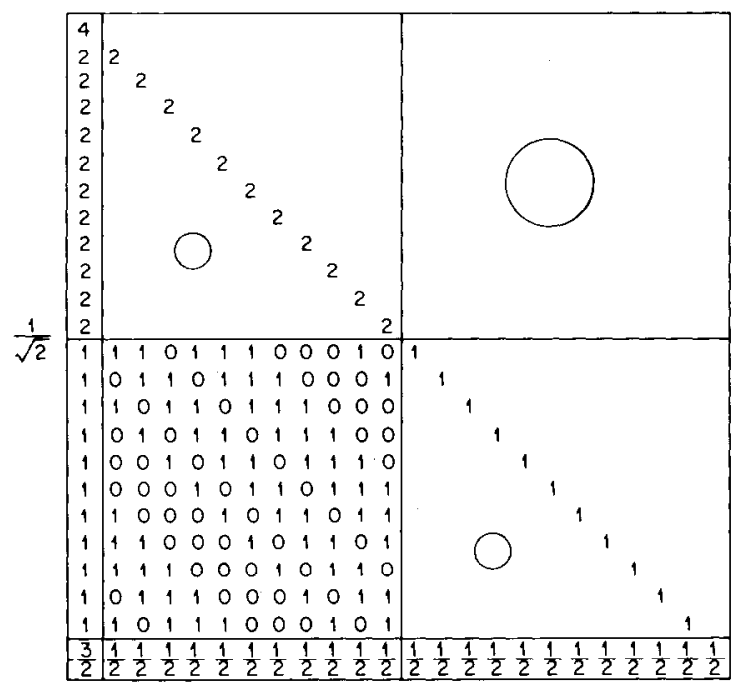

Fig. 5 Generator matrix for the 24-dimensional Leech lattice. The $11 \times$ 11 circulant matrix in the bottom left corner comes from the generator matrix of the Golay code (see [36, fig. 2.13]).

TABLE X

The LeECH LATTice IN $\boldsymbol{R}^{24}$

\begin{tabular}{|c|c|l||}
\hline$m$ & \multicolumn{1}{|c|}{$N_{m}$} & \multicolumn{1}{|c|}{ Prime Factors of $N_{m}$} \\
\hline & 1 & 1 \\
2 & 0 & 0 \\
4 & 196560 & $2^{4} \cdot 3^{3} \cdot 5 \cdot 7 \cdot 13$ \\
6 & 16773120 & $2^{12} \cdot 3^{2} \cdot 5 \cdot 7 \cdot 13$ \\
8 & 398034000 & $2^{4} \cdot 3^{7} \cdot 5^{3} \cdot 7 \cdot 13$ \\
10 & 4629381120 & $2^{14} \cdot 3^{3} \cdot 5 \cdot 7 \cdot 13 \cdot 23$ \\
12 & 34417656000 & $2^{6} \cdot 3^{3} \cdot 5^{5} \cdot 7 \cdot 13 \cdot 17 \cdot 103$ \\
14 & 187489935360 & $2^{13} \cdot 3^{7} \cdot 5 \cdot 7 \cdot 13 \cdot 23$ \\
16 & 814879774800 & $2^{4} \cdot 3^{3} \cdot 5^{2} \cdot 7 \cdot 13 \cdot 17^{2} \cdot 19 \cdot 151$ \\
18 & 2975551488000 & $2^{15} \cdot 3^{2} \cdot 5^{3} \cdot 7 \cdot 13 \cdot 887$ \\
20 & 9486551299680 & $2^{5} \cdot 3^{7} \cdot 5 \cdot 7 \cdot 13 \cdot 23 \cdot 12953$ \\
22 & 27052945920000 & $2^{12} \cdot 3^{3} \cdot 5^{4} \cdot 7 \cdot 11 \cdot 13 \cdot 17 \cdot 23$ \\
24 & 70486236999360 & $2^{6} \cdot 3^{2} \cdot 5 \cdot 7^{2} \cdot 13^{2} \cdot 59 \cdot 50093$ \\
26 & 169931095326720 & $2^{14} \cdot 3^{7} \cdot 5 \cdot 7 \cdot 13 \cdot 1489$ \\
28 & 384163586352000 & $2^{7} \cdot 3^{3} \cdot 5^{3} \cdot 7 \cdot 13 \cdot 23 \cdot 83 \cdot 5119$ \\
30 & 820166620815360 & $2^{13} \cdot 3^{3} \cdot 5 \cdot 7 \cdot 13 \cdot 17 \cdot 19 \cdot 23 \cdot 1097$ \\
32 & 1668890090322000 & $2^{4} \cdot 3^{8} \cdot 5^{3} \cdot 7 \cdot 13 \cdot 751 \cdot 1861$ \\
34 & 3249631112232960 & $2^{15} \cdot 3^{4} \cdot 5 \cdot 7 \cdot 13 \cdot 23 \cdot 116993$ \\
36 & 6096882661243920 & $2^{4} \cdot 3^{3} \cdot 5 \cdot 7^{2} \cdot 13 \cdot 17 \cdot 260654803$ \\
38 & 11045500816896000 & $2^{12} \cdot 3^{8} \cdot 5^{3} \cdot 7 \cdot 13 \cdot 23 \cdot 1571$ \\
40 & 19428439855275360 & $2^{5} \cdot 3^{3} \cdot 5 \cdot 7^{2} \cdot 13 \cdot 23 \cdot 1747 \cdot 175709$ \\
& & \\
\hline
\end{tabular}

\section{ACKNOWLEDGMENT}

I would like to thank J. H. Conway, A. Gersho, A. M. Odlyzko, and the anonymous referees for a number of helpful suggestions. The tables were computed using the Altran [7] and Macsyma [38] computer languages.

\section{REFERENCES}

[1] W. W. R. Ball and H. S. M. Coxeter, Mathematical Recreations and Essays, 12th ed. Toronto: Univ. Toronto Press, 1974.

[2] E. Bannai and N. J. A. Sloane, "Uniqueness of certain spherical codes," Canad. J. Math., 1981, to be published.

[3] E. Biglieri and M. Flia, "Cyclic-group codes for the Gaussian channel," IEEE Trans. Inform. Theory, vol. IT-22, pp. 624-629, 1976.

[4] I. F. Blake, "The Leech lattice as a code for the Gaussian channcl," Inform. Contr. vol. 19, pp. 66-74, 1971.

[5] I. F. Blake, "Distance properties of group codes for the Gaussian channel," SIAM J. Appl. Math., vol. 23, pp. 312-324, 1972.

[6] I. F. Blake and R. C. Mullin, The Mathematical Theory of Coding. New York: Academic, 1975. 
[7] W. S. Brown, Altran User's Manual, 4th ed. Murray Hill, NJ: Bell Laboratories, 1977.

[8] R. de Buda, "The upper error bound of a new near-optimal code," IEEE Trans. Inform. Theory, vol. IT-21, pp. 441-445, 1975.

[9] J. H. Conway, "A perfect group of order 8, 315, 553, 613, 086, 720, 000 , and the sporadic simple groups," Proc. Nat. Acad. Sci., vol. 61, pp. $398-400,1968$.

[10] J. H. Conway, "A group of order 8, 315, 553, 613, 086, 720, 000," Bull. London Math. S., vol. 1, pp. 79-88, 1969.

[11] J. H. Conway, "A characterization of Leech's lattice," Invent. Math., vol. 7, pp. 137-142, 1969.

[12] J. H. Conway, "Three lectures on exceptional groups," in Finite Simple Groups, M. B. Powell and G. Higman, Eds. London: Academic, 1971, pp. 215-247.

[13] H. S. M. Coxeter, "The problem of packing a number of equal nonoverlapping circles on a sphere," Trans. New York Acad. Sci., series II, vol. 24, no. 3, pp. 320-331, Jan. 1962.

[14] H. S. M. Coxeter, Regular Polytopes, 2nd ed. New York: Macmillan, 1963.

[15] H. S. M. Coxeter, Twelve Geometric Essays. Carbondale, IL: Southern Illinois Univ. Press, 1968.

[16] P. Delsarte, J. M. Goethals, and J. J. Seidel, "Spherical codes and designs," Geometriae Dedicata, vol. 6, pp. 363-388, 1977.

[17] L. E. Dickson, History of the Theory of Numbers. New York: Chelsea, 1966.

[18] A. Gersho, "Asymptotically optimal block quantization," IEEE Trans. Inform. Theory, vol. IT-25, pp. 373-380, 1979.

[19] A. Gersho, "Principles of quantization," IEEE Trans. Circuit. Syst., vol. CAS-25, pp. 427-436, July 1978.

[20] J. W. L. Glaisher; "On the numbers of representations of a number as a sum of $2 r$ squares, where $2 r$ does not exceed eighteen," Proc. London Math. Soc., series 2, vol. 5, pp. 479-490, 1907.

[21] J. M. Goethals and J. J. Seidel, "Spherical designs," in Relations Between Combinatorics and Other Parts of Mathematics, Proc. Sympos. Pure Math. XXXIV, D. K. Ray-Chaudhuri, Ed. Providence RI: Amer. Math. Soc., 1979, pp. 255-272.

[22] J. M. Goethals and J. J. Seidel, "Cubature formulae, polytopes and spherical designs," in Proc. Coxeter Symposium, Toronto, May 1979, to be published.

[23] M. Goldberg, "An improved packing of 33 equal circles on a sphere," Elemente der Mathematik, vol. 22, pp. 110-111, 1967.

[24] R. C. Gunning, Lectures on Modular Forms. Princeton: Princeton Univ. Press, 1962.

[25] G. H. Hardy, "On the representation of a number as the sum of any number of squares, and in particular of five or seven," Proc. Nat. Acad. Sci., vol. 4, pp. 189-193, 1918, (also Collected Papers, vol. I, pp. 340-344).

[26] G. H. Hardy, "On the representation of a number as the sum of any number of squares, and in particular of five," Trans. Amer. Math. Soc., vol. 21, pp. 255-284, 1920, (also Collected Papers, vol. I, pp. $345-374)$.

[27] G. H. Hardy, Ramanujan. Bronx, NY: Chelsea, 1959.

[28] G. H. Hardy and E. M. Wright, An Introduction to the Theory of Numbers, 3rd Ed. Oxford: Clarendon, 1954.

[29] G. A. Kabatiansky and V. I. Levenshtein, "Bounds for packings on a sphere and in space," Prob. Inform. Transmiss., vol. 14, no. 1, pp. $1-17,1978$.

[30] M. I. Knopp, Modular Functions in Analytic Number Theory. Chicago: Markham, 1970

[31] H. J. Landau, "How does a porcupine separate its quills?," IEEE Trans. Inform. Theory., vol. IT-17, pp. 157-161, 1971.

[32] J. Leech, "Notes on sphere packings," Canad. J. Math., vol. 19, pp. $251-267,1967$.

[33] J. Leech and N. J. A. Sloane, "New sphere packings in more than thirty-two dimensions," in Proc. Second Chapel Hill Conference on Combinatorial Mathematics and Its Applications, Chapel Hill, NC: Univ. of North Carolina, 1970, pp. 345-355.

[34] J. Leech and N. J. A. Sloane, "Sphere packings and error-correcting codes," Canad. J. Math., vol. 23, pp. 718-745, 1971.

[35] S. P. Lloyd, "Hamming association schemes and codes on spheres,"
SIAM J. Math. Anal., vol. I1,pp. 488-505, May 1980

[36]. F. J. MacWilliams and N. J. A. Sloane, The Theory of ErrorCorrecting Codes. Amsterdam; North Holland, and New York: Elsevier-North Holland, 1977.

[37] A. V. Malyšev, "On the representation of integers by positive quadratic forms with four or more variables I, Amer. Math. Soc. Translations, series 2, vol. 46, pp. 17-47, 1965.

[38] Mathlab Group, MIT, MACSYMA Reference Manual, Version 9. Cambridge MA: MIT, 1977.

[39] R. M. Mersereau, "The processing of hexagonally sampled twodimensional signals," Proc. IEEE, vol. 67, pp. 930-949, June 1979.

[40] W. C. Mitchell, "The number of lattice points in a $k$-dimensional hypersphere," Math. Comp., vol. 20, pp. 300-310, 1966.

[41] M. Newman, "A table of the coefficients of the power of $\eta(\tau)$," Proc. Kon. Ned. Akad. Wetensch., Series A, vol. 59, pp. 204-216, 1956.

[42] A. W. Nordstrom and J. P. Robinson, "An optimum nonlinear code," Inform. Contr., vol. 11, pp. 613-616, 1967.

[43] A. M. Odlyzko and N. J. A. Sloane, "New bounds on the number of unit spheres that can touch a unit sphere in $n$ dimensions," J. Comb. Theory, vol. 26A, pp. 210-214, 1979.

[44] G. Pry and I. Prigogine, "Rayons et nombres de coordination de quelques réseaux simples," Acad. Roy, Belgique, Bull. Cl. Sci. vol. 28 , pp. $866-873,1942$.

[45] H. Rademacher, Topics in Analytic Number Theory. New York: Springer, 1973.

[46] R. A. Rankin, Modular Forms and Functions. Cambridge: The University Press, 1977

[47] C. A. Rogers, Packing and Covering. Cambridge: The University Press, 1964

[48] A. Schrijver, Ed., Packing and Covering in Combinatorics, Math. Centre Tracts 106. Amsterdam: Mathematisch Centrum, 1979.

[49] L. A. Shepp, "Computerized tomography and nuclear magnetic resonance," J. Comp. Assist. Tomograph., vol. 4, no. 1, pp. 94-107, Feb. 1980.

[50] D. Slepian, "Group codes for the Gaussian channel," Bell Syst. Tech. J., vol. 47, pp. 575-602, 1968.

[51] N. J. A. Sloane, "Sphere packings constructed from BCII and Justesen codes," Mathematika, vol. 19, pp. 183-190, 1972.

[52] N. J. A. Sloane, "Binary codes, lattices, and sphere packings," in Combinatorial Surveys, P. J. Cameron, Ed. London and New York: Academic, 1977, pp. 117-164.

[53] N. J. A. Sloane, "Codes over GF(4) and complex lattices," $J$. Algebra, vol. 52, pp. 168-181, 1978.

[54] N. J. A. Sloane, "Self-dual codes and lattices," in Relations Between Combinatorics and Other Parts of Mathematics, Proc. Symp. Pure Math. XXXIV, D. K. Ray-Chaudhuri, Ed. Providence, RI: Amer. Math. Soc., 1979, pp. 273-308.

[55] N. J. A. Sloane, "A note on the Leech lattice as a code for the Gaussian channel," Inform. Contr., vol. 46, pp. 270-272, 1980.

[56] S. L. Sobolev, "Cubature formulas on the sphere invariant under finite groups of rotations," Soviet Math. Doklady, vol. 3, pp. 1307$1310,1962$.

[57] J. Tannery and I. Molk, Éléments de la théorie des Fonctions Elliptiques. 2nd Ed. Bronx, NY: Chelsea, 1972.

[58] Y. Tashiro, "On methods for generating uniform random points on the surface of a sphere," Ann. Inst. Statist. Math., vol. 29, pt. A, pp. 295-300, 1977

[59] A. Z. Val'fis, "On the representation of numbers by sums of squares - asymptotic formulas," Amer. Math. Soc. Translations, series 2, vol. 3, pp. 163-248, 1956.

[60] B. L. van der Waerden, "Pollenkörner, Punktverteilungen auf der Kugel und Informationstheorie," Die Naturwissenschaften, no. 7, pp. $189-192,1961$

[61] A. Walfisz (=A. Z. Val'fiš), "Uber Gittergunkte in mehrdimensionalen Ellipsoiden," Math. Zeit., vol. 19, pp. 300-307, 1924.

[62] E. T. Whittaker and G. N. Watson, A Course of Modern Analysis, 4th Ed. Cambridge: The University Press, 1963.

[63] A. D. Wyner, "On coding and information theory," SIAM Review, vol. 11, pp. 317-346, 1969. 